\title{
STUDY OF AN INCLINED, SEGMENTED CANTILEVER WITH TIP LOAD
}

\author{
BY \\ C. Y. WANG AND Q. DU \\ Michigan State University, East Lansing, Michigan
}

\begin{abstract}
The problem of a tip-loaded $N$-segment cantilever with torsion springs at the joints is formulated through nonlinear difference equations. The solutions are obtained by both numerical and perturbation methods. As the base is rotated the cantilever shows nonlinear behavior such as nonuniqueness, hysteresis, and catastrophic changes. A stability criterion is proposed.
\end{abstract}

Introduction. Load-bearing segmented rods occur in many situations involving both strength and flexibility. For example, the human limbs and the spine have been modelled as connected rigid rods. Partial rigidity of the system is attained by adequate support at the joints through the tendons. The mechanical analog is represented by the cantilever arm. Of great interest is the configuration of the arm under loading, since exact displacements of the tip are essential in design.

The paper is a theoretical study of a very simple model of an "arm" or cantilever. We assume there are $N$ identical rigid segments linked end to end in a plane. In the unloaded state the linked segments, with one end attached to a base, resemble a straight cantilever. There are $N$ linear torsion springs at the joints to produce a certain amount of stiffness to the system. Under a tip weight the cantilever bends as shown in Fig. 1(a). We are interested in the configuration of the cantilever as the base is rotated. The motion is assumed to be slow enough so that dynamics can be ignored.

Formulation. Let $\theta_{n}$ be the angle of the $n$th segment with the vertical (Fig. 1(b)). Let the resisting torque of the joints be proportional to the angle differences between adjacent segments with $\lambda$ as the spring constant. A moment balance gives

$$
F^{\prime} \ell \sin \theta_{n}+\lambda\left(\theta_{n+1}-\theta_{n}\right)=\lambda\left(\theta_{n}-\theta_{n-1}\right),
$$

where $F^{\prime}$ is the tip load and $\ell$ is the length of the segment. Normalize $F^{\prime}$ by $\lambda / \ell$ and drop primes. Equation (1) yields the nonlinear difference equation

$$
\theta_{n+1}-2 \theta_{n}+\theta_{n-1}+F \sin \theta_{n}=0, \quad n=1 \text { to } N .
$$

Received September 9, 1992.

1991 Mathematics Subject Classification. Primary 73G99, 73K99, 39A10. 


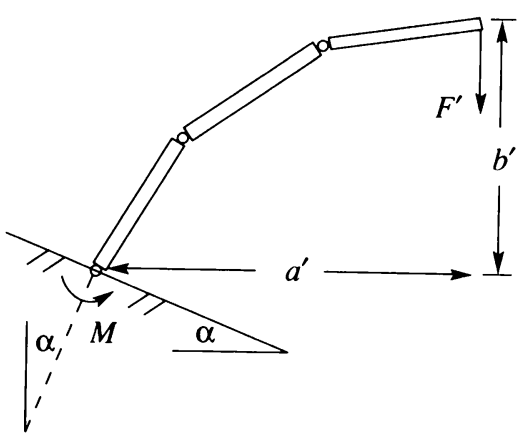

(a)

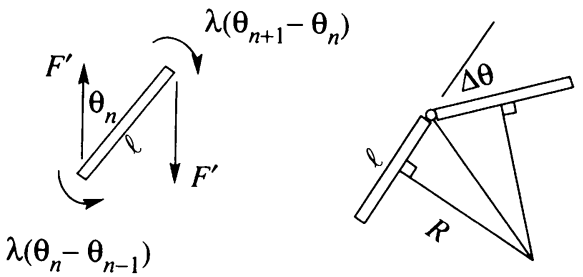

(b)

FIG. 1. (a) Cantilever with tip load. (b) Equilibrium of one segment.

(c) Radius of curvature $R$.

We also introduce two fictitious links: $n=0$, which represents the base, and $n=$ $N+1$ extending past the tip. Let $\alpha$ be the angle between the base and the horizontal. The boundary condition at the base is

$$
\theta_{0}=\alpha .
$$

Zero torque at the tip is equivalent to

$$
\theta_{N+1}=\theta_{N}
$$

For given $F, \alpha$, Eqs. (2-4) are to be solved for $\theta_{n}$. The locations of the joints are then given by

$$
\begin{array}{ll}
a_{n}=a_{n-1}+\frac{1}{N} \sin \theta_{n}, & a_{0}=0, \\
b_{n}=b_{n-1}+\frac{1}{N} \cos \theta_{n}, & b_{0}=0,
\end{array}
$$

where the lengths have been normalized by $L=\ell N$. Thus the location of the tip is

$$
\begin{aligned}
& a=a_{N}=\frac{1}{N} \sum_{n=1}^{N} \sin \theta_{n}, \\
& b=b_{N}=\frac{1}{N} \sum_{n=1}^{N} \cos \theta_{n} .
\end{aligned}
$$

The moment at the base, normalized by $\lambda$ is

$$
M=\theta_{1}-\alpha .
$$

Perturbation solution for small $F$. When the load is light or the spring is relatively stiff, $F \ll 1$. The segment angles are all close to $\alpha$. We expand in $F$ as follows:

$$
\theta_{n}=\alpha+F \varphi_{1}(n)+F^{2} \varphi_{2}(n)+F^{3} \varphi_{3}(n)+\cdots .
$$

Equations (2-4) are linearized into the successive equations

$$
\begin{aligned}
& \varphi_{1}(n+1)-2 \varphi_{1}(n)+\varphi_{1}(n-1)=-\sin \alpha, \\
& \varphi_{2}(n+1)-2 \varphi_{2}(n)+\varphi_{2}(n-1)=-\cos \alpha \varphi_{1}(n), \\
& \varphi_{3}(n+1)-2 \varphi_{3}(n)+\varphi_{3}(n-1)=\frac{\sin \alpha}{2} \varphi_{1}^{2}(n)-\cos \alpha \varphi_{2}(n),
\end{aligned}
$$




$$
\begin{aligned}
\varphi_{i}(0) & =0, \\
\varphi_{i}(N) & =\varphi_{i}(N+1), \quad i \text { integer. }
\end{aligned}
$$

The solutions are polynomials

$$
\begin{gathered}
\varphi_{1}(n)=-\frac{\sin \alpha}{2}\left[n^{2}-(2 N+1) n\right] \\
\varphi_{2}(n)=\frac{\cos \alpha \sin \alpha}{24}\left[n^{4}-(4 N+2) n^{3}-n^{2}\right. \\
\left.\quad+\left(8 N^{3}+12 N^{2}+8 N+2\right) n\right], \\
\varphi_{3}(n)=\frac{\sin ^{3} \alpha}{240}\left[n^{6}-(6 N+3) n^{5}+\left(10 N^{2}+10 N\right) n^{4}\right. \\
\quad+(10 N+5) n^{3}-\left(10 N^{2}+10 N+1\right) n^{2} \\
\left.\quad-\left(16 N^{5}+40 N^{4}+40 N^{3}+20 N^{2}+8 N+2\right) n\right] \\
+\frac{\cos ^{2} \alpha \sin \alpha}{720}\left[-n^{6}+(6 N+3) n^{5}+5 n^{4}\right. \\
\quad-\left(40 N^{3}+60 N^{2}+50 N+15\right) n^{3}-4 n^{2} \\
\left.\quad+\left(96 N^{5}+240 N^{4}+280 N^{3}+180 N^{2}+68 N+12\right) n\right] .
\end{gathered}
$$

Higher-order solutions can be generated with the help of a computer with symbolic computation capability. The moment at the base is then

$$
\begin{aligned}
M=N \sin \alpha F[1+ & \frac{1}{6}(N+1)(2 N+1) \cos \alpha F \\
& +\frac{1}{60}(N+1)(2 N+1)\left(2 N^{2}+2 N+1\right)\left(2 \cos ^{2} \alpha-\sin ^{2} \alpha\right) F^{2} \\
& \left.+O\left(N^{2} F\right)^{3}\right] .
\end{aligned}
$$

The horizontal distance of the tip is $a=M /(F N)$.

Perturbation when $\alpha$ and $\theta$ are close to $\pi$. In this case the cantilever is hanging almost vertically down. Let $\alpha=\pi+\varepsilon, \varepsilon \ll 1$. We expand as follows:

$$
\theta_{n}=\pi+\varepsilon \chi_{1}(n)+\varepsilon^{2} \chi_{2}(n)+\varepsilon^{3} \chi_{3}(n)+\cdots
$$

Equation (2) becomes the successive linear difference equations

$$
\begin{array}{r}
\chi_{1}(n+1)-(F+2) \chi_{1}(n)+\chi_{1}(n-1)=0, \\
\chi_{1}(0)=1, \quad \chi_{1}(N)=\chi_{1}(N+1), \\
\chi_{2}(n+1)-(F+2) \chi_{2}(n)+\chi_{2}(n-1)=0, \\
\chi_{2}(0)=0, \quad \chi_{2}(N)=\chi_{2}(N+1), \\
\chi_{3}(n+1)-(F+2) \chi_{3}(n)+\chi_{3}(n-1)=-\frac{1}{6} \chi_{1}^{3}(n), \\
\chi_{3}(0)=0, \quad \chi_{3}(N)=\chi_{3}(N+1) .
\end{array}
$$

The general solution for $\chi_{1}(n)$ is

$$
\chi_{1}(n)=B_{1} \nu_{1}^{n}+B_{2} \nu_{2}^{n}
$$


where

$$
\nu_{1,2}=1+\frac{F}{2} \pm \frac{1}{2} \sqrt{F^{2}+4 F} .
$$

Using the boundary conditions we find

$$
\begin{gathered}
B_{1}=\nu_{2}^{N}\left(\nu_{2}-1\right) / D, \quad B_{2}=\nu_{1}^{N}\left(1-\nu_{1}\right) / D, \\
D \equiv \nu_{1}^{N}\left(1-\nu_{1}\right)-\nu_{2}^{N}\left(1-\nu_{2}\right) .
\end{gathered}
$$

From Eqs. $(23,24)$ we conclude that $\chi_{2}$ is identically zero. The solution $\chi_{3}(n)$ is more complicated. The right side of Eq. (25) is expressed as

$$
-\frac{1}{6}\left[B_{1}^{3}\left(\nu_{1}^{3}\right)^{n}+3 B_{1}^{2} B_{2} \nu_{1}^{n}+3 B B_{2}^{2} \nu_{2}^{n}+B_{2}^{3}\left(\nu_{2}^{3}\right)^{n}\right] \text {. }
$$

By a parameter variation method similar to that described in Mickens [1] the particular solution is

$$
\begin{array}{r}
\chi_{3 p}(n)=-\frac{1}{6}\left\{\frac{\left(B_{1} \nu_{1}^{n+1}\right)^{3}}{\nu_{1}^{6}-(F+2) \nu_{1}^{3}+1}+\frac{\left(B_{2} \nu_{2}^{n+1}\right)^{3}}{\nu_{2}^{6}-(F+2) \nu_{2}^{3}+1}\right. \\
\left.+\frac{3 B_{1}^{2} B_{2}}{\nu_{1}^{2}-1} n \nu_{1}^{n+1}+\frac{3 B_{2}^{2} B_{1}}{\nu_{2}^{2}-1} n \nu_{2}^{n+1}\right\} .
\end{array}
$$

Using the boundary conditions and a homogeneous solution similar to Eq. (27) we find

$$
\begin{aligned}
\chi_{3}(n)=\chi_{3 p}(n)+\frac{1}{D}\left\{\left[\chi_{3 p}(0) \nu_{2}^{N}\left(1-\nu_{2}\right)+\chi_{3 p}(N+1)-\chi_{3 p}(N)\right] \nu_{1}^{n}\right. \\
\left.-\left[\chi_{3 p}(0) \nu_{1}^{N}\left(1-\nu_{1}\right)+\chi_{3 p}(N+1)-\chi_{3 p}(N)\right] \nu_{2}^{n}\right\} .
\end{aligned}
$$

The moment at the base is then

$$
M=\left[\chi_{1}(1)-1\right] \varepsilon+\chi_{3}(1) \varepsilon^{3}+O\left(\varepsilon^{5}\right) .
$$

Perturbation when $\alpha$ and $\theta$ are small. This is the almost vertically standing cantilever. We expand as follows:

$$
\theta_{n}=\alpha \psi_{1}(n)+\alpha^{3} \psi_{3}(n)+O\left(\alpha^{5}\right)
$$

Similarly, the leading equations are

$$
\begin{gathered}
\psi_{1}(n+1)+(F-2) \psi_{1}(n)+\psi_{1}(n-1)=0, \\
\psi_{1}(0)=1, \quad \psi_{1}(N)=\psi_{1}(N+1) .
\end{gathered}
$$

The solution is

$$
\psi_{1}(n)=C_{1} \mu_{1}^{n}+C_{2} \mu_{2}^{n}
$$

where

$$
\mu_{1,2} \equiv 1-\frac{F}{2} \pm \frac{1}{2} \sqrt{F^{2}-4 F}, \quad F \neq 4 .
$$

Note that $\mu_{1,2}$ are complex conjugates if $F<4$, indicating oscillatory solutions. The boundary conditions give

$$
C_{1}=\mu_{2}^{N}\left(\mu_{2}-1\right) / D^{\prime}, \quad C_{2}=\mu_{1}^{N}\left(1-\mu_{1}\right) / D^{\prime},
$$




$$
D^{\prime} \equiv \mu_{1}^{N}\left(1-\mu_{1}\right)-\mu_{2}^{N}\left(1-\mu_{2}\right) \text {. }
$$

The equation for $\psi_{3}(n)$ is

$$
\begin{gathered}
\psi_{3}(n+1)+(F-2) \psi_{3}(n)+\psi_{3}(n-1)=\frac{1}{6} \psi_{1}^{3}(n), \\
\psi_{3}(0)=0, \quad \psi_{3}(N)=\psi_{3}(N+1)
\end{gathered}
$$

The solution is

$$
\begin{gathered}
\psi_{3 p}(n)=-\frac{1}{6}\left\{\frac{\left(C_{1} \mu_{1}^{n+1}\right)^{3}}{\mu_{1}^{6}+(F-2) \mu_{1}^{3}+1}+\frac{\left(C_{2} \mu_{2}^{n+1}\right)^{3}}{\mu_{2}^{6}+(F-2) \mu_{2}^{3}+1}\right. \\
\left.+\frac{3 C_{1}^{2} C_{2}}{\mu_{1}^{2}-1} n \mu_{1}^{n+1}+\frac{3 C_{2}^{2} C_{1}}{\mu_{2}^{2}-1} n \mu_{2}^{n+1}\right\}, \\
\psi_{3}(n)=\psi_{3 p}(n)+\frac{1}{D^{\prime}}\left\{\left[\psi_{3 p}(0) \mu_{2}^{N}\left(1-\mu_{2}\right)+\psi_{3 p}(N+1)-\psi_{3 p}(N)\right] \mu_{1}^{n}\right. \\
\left.-\left[\psi_{3 p}(0) \mu_{1}^{N}\left(1-\mu_{1}\right)+\psi_{3 p}(N+1)-\psi_{3 p}(N)\right] \mu_{2}^{n}\right\} .
\end{gathered}
$$

In the case $F=4$, we find $\mu_{2}=\mu_{1}=-1$,

$$
\begin{gathered}
\psi_{1}(n)=(-1)^{n}-\frac{2}{2 N+1} n(-1)^{n}, \\
\psi_{3 p}(n)=\frac{n^{2}(-1)^{n}}{6}\left[-\frac{1}{2}+\frac{n}{2 N+1}-\frac{\left(n^{2}-1\right)}{(2 N+1)^{2}}+\frac{2}{5(2 N+1)^{3}} n\left(n^{2}-\frac{5}{3}\right)\right], \\
\psi_{3}(n)=\psi_{3 p}(n)+\frac{\psi_{3 p}(N+1)-\psi_{3 p}(N)}{(-1)^{N}(2 N+1)} n(-1)^{n} .
\end{gathered}
$$

The moment at the base is then

$$
M=\left[\psi_{1}(1)-1\right] \alpha+\psi_{3}(1) \alpha^{3}+O\left(\alpha^{5}\right) .
$$

Stability problem when $\alpha=0$. The stability of a linked column under axial compression is of great interest to structural engineers (Croll and Walker [2], Weinberger [3], Simitses [4]). Their analyses, however, are for the columns with one or two degrees of freedom only. We shall study the stability of the $N$-link cantilever-column.

The stability criterion can be obtained from Eq. (35). For $\alpha=0$ and $\theta_{n} \neq 0$, a necessary condition resulting from the previous analysis is $D^{\prime}=0$, or

$$
\mu_{1}^{N}\left(1-\mu_{1}\right)-\mu_{2}^{N}\left(1-\mu_{2}\right)=0 .
$$

Since $\mu_{1}, \mu_{2}$ are related by

$$
\mu_{1} \mu_{2}=1, \quad \mu_{1}+\mu_{2}=2-F,
$$

Eq. (50) simplifies to

$$
\mu_{1}^{2 N+1}=-1
$$

The solution is

$$
\mu_{1,2}=e^{ \pm i \pi \frac{(2 m-1)}{(2 N+1)}}, \quad m=1,2, \ldots, 2 N+1
$$


From Eq. (51) the critical force is

$$
F_{\mathrm{c}}=2-2 \cos \left[\frac{\pi(2 m-1)}{(2 N+1)}\right]=4 \sin ^{2}\left[\frac{\pi(2 m-1)}{2(2 N+1)}\right] .
$$

Now as $N \rightarrow \infty$ we expect the segmented cantilever to approach a continuous elastic cantilever. Let us define a local "radius of curvature" $R$ as shown in Fig. $1(\mathrm{c})$. Thus

$$
\tan \frac{\Delta \theta}{2}=\frac{\ell}{2 R} \text {. }
$$

If local moment is defined as stiffness " $E I$ " times local curvature,

$$
\lambda \Delta \theta=E I \frac{2}{\ell} \tan \frac{\Delta \theta}{2} .
$$

We see that for a segmented rod with linear torsion springs the bending stiffness $E I$ decreases as $\Delta \theta$ increases or the material is "soft". As $N \rightarrow \infty, \Delta \theta \rightarrow 0$ and we find

$$
E I \approx \lambda \ell=\lambda L / N .
$$

The critical leads for a fixed-free elastic column (Timoshenko and Gere [5]) are

$$
\frac{F^{\prime} L^{2}}{E I}=\frac{(2 m-1)^{2} \pi^{2}}{4}, \quad m \text { integer. }
$$

Using Eq. (57) the asymptotic value of our normalization as $N \rightarrow \infty$ is

$$
F=\frac{F^{\prime} \ell}{\lambda}=\frac{(2 m-1)^{2} \pi^{2}}{4 N^{2}}
$$

Comparing the numerical values of Eqs. (54) and (59) we see that the difference in critical force between the continuous elastic column and the discrete segmented column is within $5 \%$ if $N>19$. For a difference of $3 \%$ the number of segments needs to be larger than 32 .

Numerical solution. When neither $F$ nor $\theta_{i}$ is small, the problem is basically nonlinear and numerical solution is necessary. The method is as follows. For given $N, \alpha$ and $F>F_{\text {c }}$ we guess $\theta_{1}$ and compute $\theta_{N}, \theta_{N+1}$ from Eq. (2). A solution is found when Eq. (4) is satisfied. If not, the value of $\theta_{1}$ is adjusted.

The nonlinear problem can best be depicted in a base moment-base angle plot. We shall illustrate by the $N=6$ case. Figure 2 shows the $(M, \alpha)$-plot for $F=0.05$. Since the value of $F$ is less than the lowest buckling force $F_{\mathrm{c}}=0.0581$ from Table 1, we expect $M$ to be a unique function of $\alpha$. The maximum $M$ of 0.2901 (or longest horizontal reach of the cantilever occurs at $\alpha=49^{\circ}$. Our approximate solution Eq.

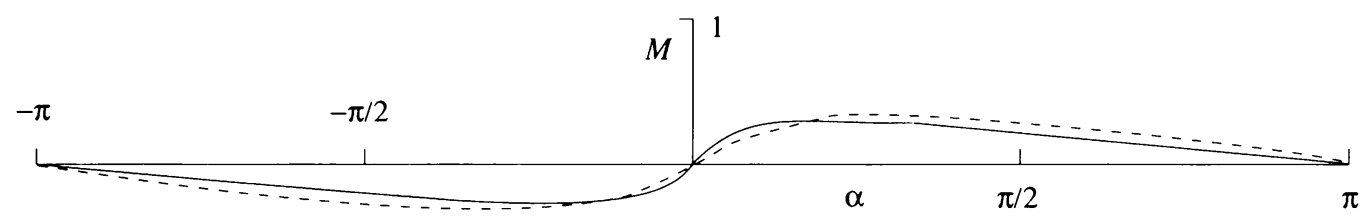

Fig. 2. Moment $M$ and angle $\alpha$ at base, $N=6, F=0.05$.

Dashed line is from Eq. (19). 


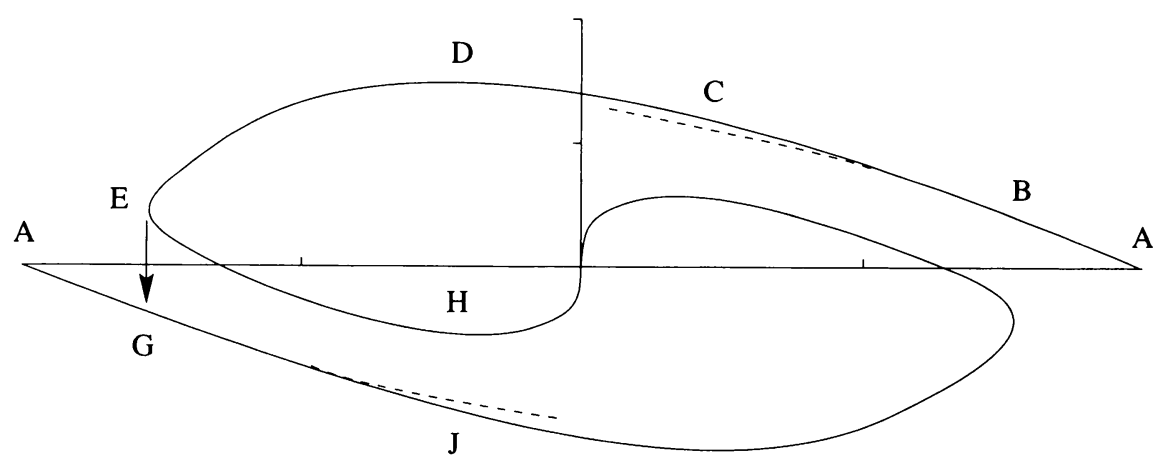

Fig. 3. $(M, \alpha)$-diagram for $N=6, F=0.5$. Dashed line is from Eq. (34).

(19) for small $F$ compares well with the numerically exact solution for all $\alpha$.

When $F$ is increased to 0.5 (Fig. 3), nonuniqueness is already apparent. There are three solutions for $|\alpha|<138^{\circ}$ and one solution elsewhere. Our perturbation solution for $\alpha$ close to $\pi$, Eq. (34), is surprisingly accurate. At state A, $\alpha=180^{\circ}$ and the cantilever is hanging straight down. As $\alpha$ is decreased the shapes change continuously through states B, C, D, E till $\alpha=-138^{\circ}$. Any further decrease would cause a flipping phenomena from states $\mathrm{E}$ to $\mathrm{G}$. The corresponding configurations are shown in Fig. 4(a). The three equilibrium solutions (not all stable) for $\alpha=-40^{\circ}$ are shown in Fig. 4(b).

Figure 5 (see p. 88) shows the $(M, \alpha)$-diagram for $F=1$. For given $\alpha$ there may be one, three, or five solutions. Notice the folding of the curve near $\alpha= \pm \pi$. For $F=1.5$ the foldings become more complicated (Fig. 6 on p. 88). There may be as many as 11 different solutions.

Figure 7 (see p. 89) shows a composite picture of several $(M, \alpha)$ diagrams for $N=3$. The behavior is similar and nonlinearity persists even for a small number of segments.

Of interest is the force-displacement relation for the vertical segmented column $(\alpha=0)$. Figure 8 (see p. 89) shows the location of the tip for $N=3$. As $F$ is increased from zero, the column remains straight until $F$ reaches the first critical force of 0.1981 .

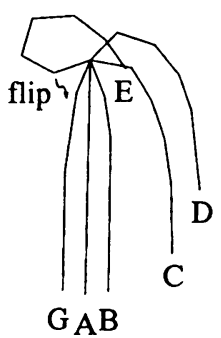

(a)

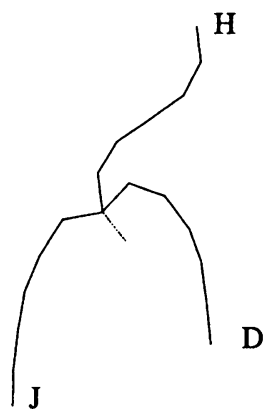

(b)

Fig. 4. (a) Configurations as $\alpha$ is decreased. Letters correspond to states in Fig. 3. (b) Three different solutions for $\alpha=-40^{\circ}, N=6$, $F=0.5$. 


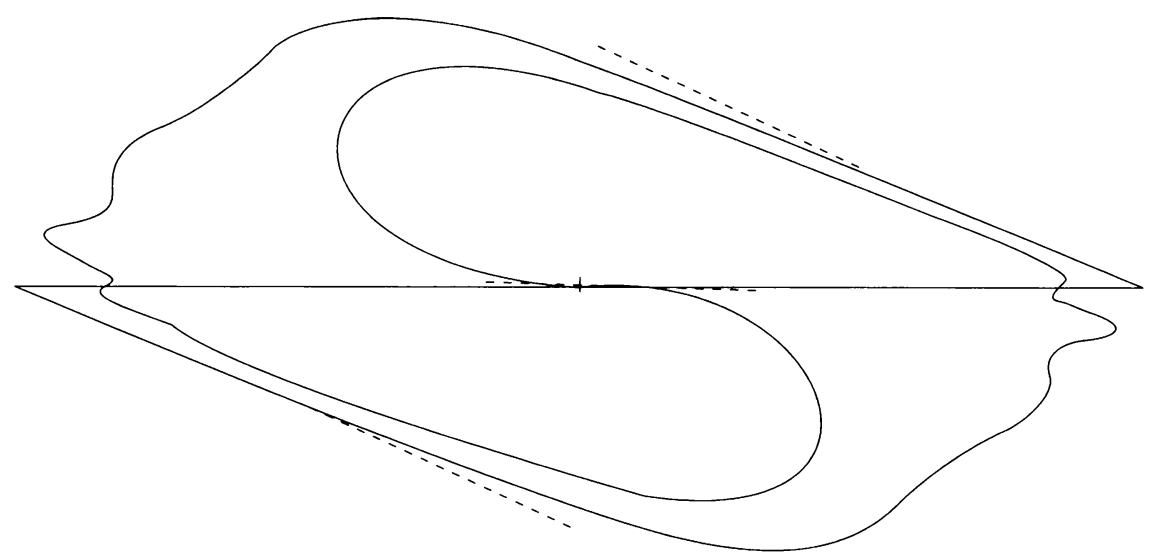

Fig. 5. $(M, \alpha)$-diagram for $N=6, F=1$. Approximations are from Eqs. (34) or (49).

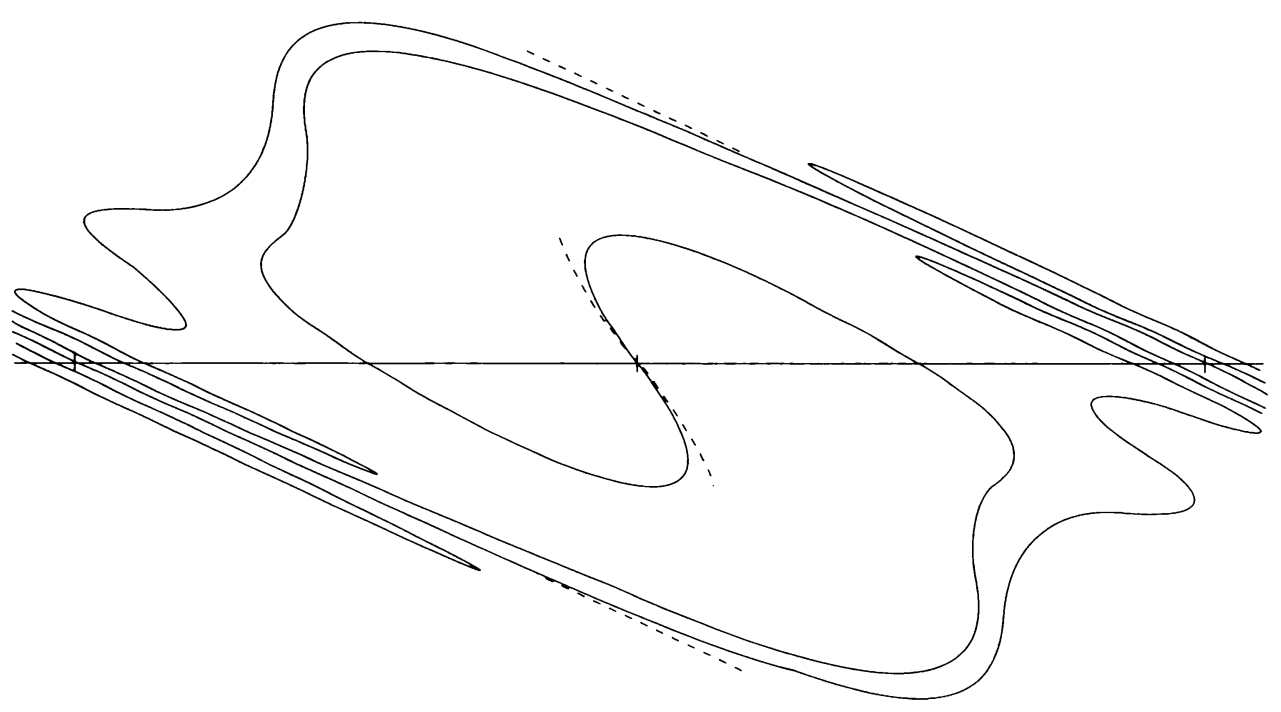

Fig. 6. $(M, \alpha)$-diagram for $N=6, F=1.5$. Approximations are from Eqs. (34) or (49).

Then the vertical distance $b$ decreases sharply from 1 and is asymptotic to -1 for large $F$. The horizontal distance quickly rises to a maximum (longest reach) then gradually decreases to zero. The second mode bifurcates at $F=1.555$ with a gentler slope. Figure 9 (see p. 90) shows the results for $N=6$. For the range considered, three modes are possible. 


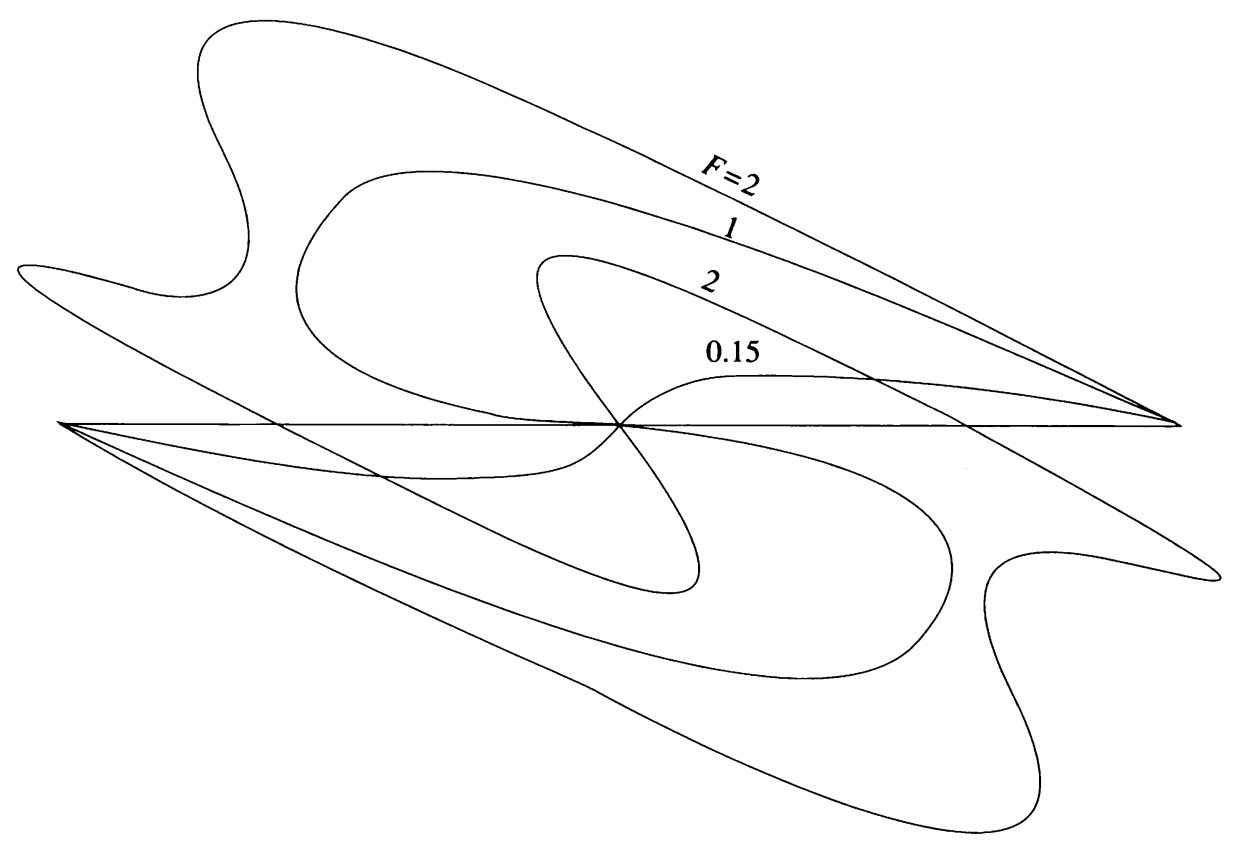

Fig. 7. $(M, \alpha)$-diagrams for $N=3$.

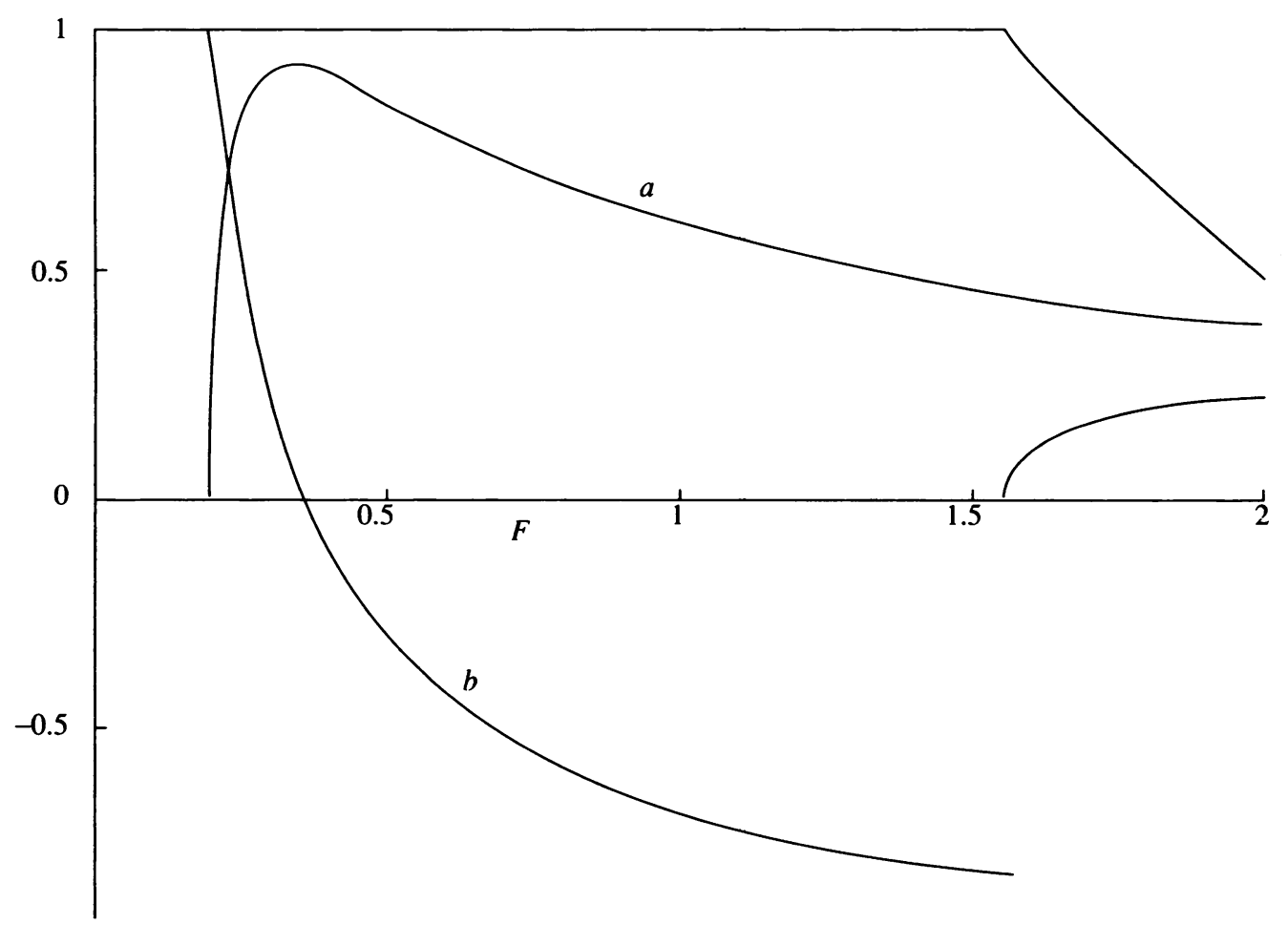

Fig. 8. Tip displacements versus $F$ for $N=3$. 


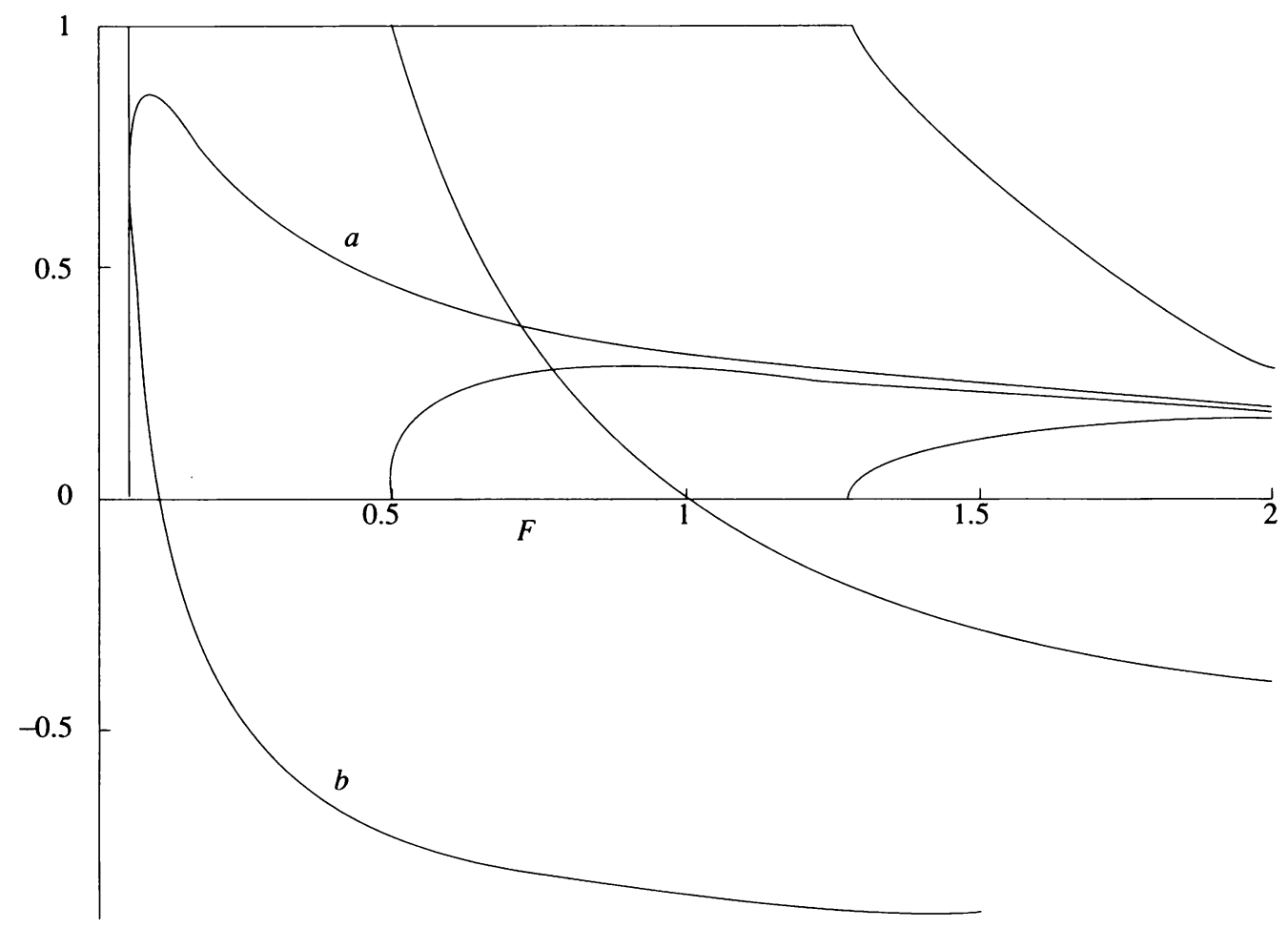

Fig. 9. Tip displacements versus $F$ for $N=6$.

Some uniqueness observations. Let $\mathrm{A}$ be the $N \times N$ matrix

$$
\mathbf{A}=\left(\begin{array}{cccccc}
-2 & 1 & & & & \\
1 & -2 & 1 & & & \\
& 1 & -2 & 1 & & \\
& & \ddots & \ddots & \ddots & \\
& & & & -2 & 1 \\
& & & & 1 & -1
\end{array}\right)
$$

and $\theta$ be the vector $\left(\theta_{1}, \theta_{2}, \ldots, \theta_{N}\right)^{\mathrm{T}}$. When $\alpha=0$ Eqs. (2-4) linearize to

$$
(\mathbf{A}+F \mathbf{I}) \boldsymbol{\theta}=0 .
$$

The eigenvalues $F_{\mathrm{c}}$ are given by Eq. (54). Let $F_{1}$ be the lowest eigenvalue $4 \sin ^{2}[\pi /(4 N+2)]$.

Now for given general $\theta_{0}=\alpha, \theta_{N+1}=\theta_{N}$, Eqs. (2-4) are equivalent to

$$
\mathbf{G}(\boldsymbol{\theta}, F)=0 \text {, }
$$

where

$$
\mathbf{G}=\left(G_{1}(\boldsymbol{\theta}, F), G_{2}(\boldsymbol{\theta}, F), \ldots, G_{N}(\boldsymbol{\theta}, F)\right)
$$

and

$$
G_{k}=\theta_{k+1}-2 \theta_{k}+\theta_{k-1}+F \sin \theta_{k}
$$


Bifurcation or nonuniqueness occurs when

$$
\operatorname{det}\left(\frac{\partial \mathbf{G}}{\partial \theta}\right)=\operatorname{det} \mathbf{D}=0
$$

where $\mathbf{B}$ is the diagonal matrix with elements $\left\{\cos \theta_{1}, \ldots, \cos \theta_{N}\right\}$ and $\mathbf{D}=\mathbf{A}+F \mathbf{B}$.

We can deduce the following results:

(a) If $F<F_{1}$, for all $\alpha$ the solution $\theta$ is unique.

Since $\mathbf{A}+F \mathbf{I}$ is now negative definite and $F(\mathbf{B}-\mathbf{I})$ is negative semidefinite, thus $\mathbf{D}=(\mathbf{A}+F \mathbf{I})+F(\mathbf{B}-\mathbf{I})$ is negative definite. Therefore Eq. (65) has no solution and the solution $\boldsymbol{\theta}$ is unique. This case is represented by Fig. 2.

(b) For $F$ sufficiently small but $\alpha$ close to $\pm \pi$, the solution is unique.

For $F$ sufficiently small, we expect $\theta_{k}$ is close to $\pi$, even if $F>F_{1}$. Thus $F \mathbf{B}$ is close to $-F \mathbf{I}$ and det $\mathbf{D}$ will never be zero. This case is represented by Fig. 3 .

The stability of the equilibrium solutions. Figure 6 shows a multitude of equilibrium solutions for given $F$ and $\alpha$. Some may be unstable to small perturbations and therefore cannot be realized in practice. In general, let the tip be displaced from the equilibrium position by a small amount $(\xi, \eta)$. The solution is stable if the induced reactive force of the cantilever tends to return the tip to its original equilibrium position.

Let tip forces $(-X,-Y)$ be applied to a given equilibrium solution $\alpha, F, \theta$. A moment balance on a segment similar to Fig. 1(c) gives

$$
\begin{aligned}
& \left(\theta_{n+1}+\delta_{n+1}\right)-2\left(\theta_{n}+\delta_{n}\right)+\left(\theta_{n-1}+\delta_{n-1}\right) \\
& \quad+(F+Y) \sin \left(\theta_{n}+\delta_{n}\right)-X \cos \left(\theta_{n}+\delta_{n}\right)=0
\end{aligned}
$$

where $\boldsymbol{\delta}=\left(\delta_{1}, \ldots, \delta_{N}\right)^{\mathrm{T}}$ is the perturbation on $\boldsymbol{\theta}$. Equation (66) linearizes to

$$
\mathbf{D} \boldsymbol{\delta}+Y \mathbf{C e}-X \mathbf{B e}=0 .
$$

Here $\mathbf{e}=(1, \ldots, 1)^{\mathrm{T}}$ and $\mathbf{C}$ is the $N \times N$ diagonal matrix with elements $\left(\sin \theta_{1}, \ldots\right.$, $\left.\sin \theta_{N}\right)$. Equations $(7,8)$ linearize to

$$
\xi=\frac{1}{N} \mathbf{e}^{\mathrm{T}} \mathbf{B} \boldsymbol{\delta}, \quad \eta=\frac{-1}{N} \mathbf{e}^{\mathrm{T}} \mathbf{C} \boldsymbol{\delta} .
$$

We assume $(\mathbf{A}+F \mathbf{B})$ is nonsingular and the transformation $(\xi, \eta) \rightarrow(X, Y)$ is one to one. Then a potential $\Omega$ exists such that

$$
\nabla \Omega=-(X, Y) \text {. }
$$

The solution $\alpha, F, \theta$ is stable if $\Omega$ is convex. This is equivalent to the following theorem:

ThEOREM. An equilibrium solution is stable if and only if $\left(\begin{array}{cc}u & -w \\ -w & v\end{array}\right)$ is negative definite, where

$$
\begin{gathered}
u=\frac{1}{N} \mathbf{e}^{\mathrm{T}} \mathbf{B D}^{-1} \mathbf{B e}, \quad v=\frac{1}{N} \mathbf{e}^{\mathrm{T}} \mathbf{C D}^{-1} \mathbf{C e}, \\
w=\frac{1}{N} \mathbf{e}^{\mathrm{T}} \mathbf{C D}^{-1} \mathbf{B e}=\frac{1}{N} \mathbf{e}^{\mathrm{T}} \mathbf{B D}^{-1} \mathrm{Ce} .
\end{gathered}
$$


Proof. $\Omega$ is convex if and only if the Jacobian $(\partial(X, Y) / \partial(\xi, \eta))$ is negative definite or equivalently $(\partial(\xi, \eta) / \partial(X, Y))$ is negative definite. From Eq. (67) we find

$$
\mathbf{D} \frac{\partial \boldsymbol{\delta}}{\partial X}=\mathbf{B e}, \quad \mathbf{D} \frac{\partial \boldsymbol{\delta}}{\partial Y}=-\mathbf{C e} .
$$

Therefore Eq. (68) yields

$$
\frac{\partial \xi}{\partial X}=\frac{1}{N} \mathbf{e}^{\mathrm{T}} \mathbf{B} \frac{\partial \boldsymbol{\delta}}{\partial X}=\frac{1}{N} \mathbf{e}^{\mathrm{T}} \mathbf{B D}{ }^{-1} \mathbf{B e}=u .
$$

Similarly

$$
\frac{\partial \xi}{\partial Y}=-w, \quad \frac{\partial \eta}{\partial X}=-w, \quad \frac{\partial \eta}{\partial Y}=v .
$$

Corollary. If $\mathbf{D}$ is negative definite for some equilibrium solution and $\mathrm{Be}, \mathrm{Ce}$ are not parallel to each other, then the solution is stable.

Proof. Since $\mathbf{D}^{-1}$ is also negative definite and $\mathbf{B e}$, Ce are not parallel, we have

$$
u=\frac{1}{N}(\mathbf{B e})^{\mathrm{T}} \mathbf{D}^{-1}(\mathbf{B e})<0, \quad v=\frac{1}{N}(\mathbf{C} \mathbf{e})^{-1} \mathbf{D}^{-1}(\mathbf{C} \mathbf{e})<0 .
$$

Also, from the symmetry and negative definiteness of $\mathbf{D}^{-1}$, one has

$$
w^{2}=\left[(\mathbf{C e})^{\mathrm{T}} \mathbf{D}^{-1}(\mathbf{B e})\right]^{2}<\left[(\mathbf{C e})^{\mathrm{T}} \mathbf{D}^{-1}(\mathbf{C e})\right]\left[(\mathbf{B e})^{\mathrm{T}} \mathbf{D}^{-1}(\mathbf{B e})\right]=u v .
$$

Thus $((u,-w),(-w, v))$ is negative definite and the solution is stable.

Using the theorem, one can easily check the stability of each equilibrium solution. For example, the solution $H$ in Fig. 4(b) is not stable, while solutions $J$ and $D$ are both stable. In general, in the moment-angle diagrams of Figs. (5-7), if the matrix D is negative definite at some point on a branch, then the whole branch between its bifurcation points contains only stable solutions. On the other hand, for a branch on which $\mathbf{D}$ is nowhere negative definite, we may have both stable and unstable solutions.

Discussion. Physically the segmented cantilever is similar to the elastica cantilever studied by Wang [6]. Both types of problems show high degree of nonlinearity at large $F$, which is exemplified by the phenomena of flipping, hysteresis, bifurcation, and nonuniqueness. We emphasize here that the moment-angle diagram is important in characterizing nonlinearities.

The buckling load for the discrete case is comparable to the continuous case if the number of segments is larger than 32 . The same cannot be said about the postbuckling configurations. Even for large $N$, some local angle changes may be large. Since the segmented cantilever, having a softening "stiffness", is much more pliable at larger angle differences, the tip location may be quite different from that predicted by the elastica theory.

We considered linear torsion springs in order to isolate the effects of geometric nonlinearity reflected in this paper. If in addition the springs are nonlinear, a Taylor expansion would add some terms proportional to the cube of the angle differences in Eq. (1). The stability results will not be affected while the solutions for small $F$ will change slightly. The large $F$ solutions will be greatly altered.We expect the nonlinear phenomena to be much diminished due to the added stiffness. 
Mathematically the segmented cantilever is governed by nonlinear difference equations, the solution of which differs from differential equations. We find regular perturbations on the difference equations can be successfully performed. However, singular perturbation (for large $F$ ) fails. This is probably because discrete segments do not admit similarity stretching transforms which are necessary for a boundary layer.

\section{REFERENCES}

[1] R. E. Mickens, Difference Equations, 2nd ed., Van Nostrand, New York, 1990

[2] J. G. Croll and A. C. Walker, Elements of Structural Stability, Wiley, New York, 1972

[3] H. F. Weinberger, Variational Methods for Eigenvalue Approximation, SIAM, Philadelphia, 1974

[4] G. J. Simitses, Elastic Stability of Structures, Prentice Hall, Englewood Cliffs, New Jersey, 1976

[5] S. P. Timoshenko and J. M. Gere, Theory of Elastic Stability, McGraw-Hill, New York, 1961

[6] C. Y. Wang, Large deflections of an inclined cantilever with an end load, Internat J. Non-Linear Mech. 16, 155-164 (1981) 